\title{
FATORES DE INFLUÊNCIA NA ADESÃO E NA RESISTÊNCIA MECÂNICA DE PEÇAS POLIMÉRICAS SOLDADAS POR ATRITO ROTACIONAL: UMA REVISÃO DA LITERATURA
}

\author{
Ricardo Coleoni Borges ${ }^{*}$, Daniela Lupinacci Villanoval, Edson Francisquetti ${ }^{1}$ \\ 1 Programa de Pós-Graduação em Tecnologia e Engenharia de Materiais - PPGTEM, Instituto Federal do Rio Grande do Sul, \\ Multicampi, Brasil.
}

*E-mail:rrcoleoni@gmail.com

\begin{abstract}
RESUMO
$\mathrm{O}$ uso de materiais poliméricos na indústria tem ganhado cada vez mais espaço. Devido à sua aplicabilidade, a união de diversos componentes é comumente necessária. Métodos usuais de união de polímeros são fixadores mecânicos, adesivos e processos de soldagem polimérica. Na união por soldagem por atrito rotacional, o calor é gerado por intermédio de movimento mecânico (friç̧ão) interno, fundindo ou amolecendo o polímero na região da interface, propiciando difusão intermolecular e emaranhamento das cadeias. Para melhor compreensão dos fenômenos que influenciam na resistência de peças poliméricas soldadas, realizou-se uma revisão da literatura por meio de levantamento bibliográfico nos bancos de dados Science Direct, Portal de Periódicos da Capes e Google Scholar com critérios pré-definidos. Constatou-se que as etapas de maior influência na resistência mecânica de juntas soldadas são as de aquecimento e resfriamento, além de outros aspectos como utilização adequada de perfis de solda, uso de materiais com propriedades bem dimensionadas ao processo e peças com bom acabamento superficial (sem a presença de aditivos, contaminantes ou outros).
\end{abstract}

Palavras-chave: Soldagem. Polímeros. Atrito-Rotacional.

\section{Introdução}

$\mathrm{O}$ uso de materiais poliméricos na indústria tem ganhado cada vez mais espaço em decorrência do grande número de variações disponíveis que propiciam flexibilidade na escolha por melhores propriedades ópticas, químicas, estruturais ou processuais para cada aplicação, aliadas ao baixo investimento financeiro, comparativamente a outros materiais com propriedades similares.

Devido à sua aplicabilidade, a união de diversos componentes é comumente necessária, dadas as restrições técnicas e econômicas em produzir peças de excessivo tamanho e complexidade para a moldagem em uma única operação e pela necessidade em unir materiais diferentes em uma mesma peça [1]. Métodos usuais de união de polímeros são fixadores mecânicos, adesivos e processos de soldagem polimérica.

$\mathrm{Na}$ aplicação de fixadores mecânicos a união é realizada através da aplicação de parafusos, rebites, engates moldados ou outros fixadores [2]. As juntas, porém, não apresentam bom desempenho mecânico devido à concentração de tensões gerada na região, no entorno do fixador, podendo levar à formação de trincas e falhas [3].

$\mathrm{Na}$ aplicação de adesivos, um material extra é aplicado na linha de junção para unir ambas as partes, sendo uma técnica vantajosa para a união de diferentes materiais. Entretanto, sua aplicação demanda uma etapa de cura do adesivo, tornando o processo longo e caro e resultando em um produto final com restrições a ambientes hostis ou com temperaturas elevadas [4].

$\mathrm{Na}$ união por soldagem, o calor é utilizado para fundir ou amolecer o polímero na região da interface, propiciando difusão intermolecular e emaranhamento das cadeias [1]. Uniões produzidas por soldagem, se bem executadas, são capazes de gerar peças com propriedades mecânicas idênticas, ou mesmo superiores à de peças produzidas em uma única operação, em virtude do acúmulo de material sobressalente que reforça a linha de solda em algumas operações.

Dentre as técnicas de soldagem disponíveis, o processo de soldagem por atrito rotacional é de grande interesse para a indústria de processamento de polímeros devido a seus baixos tempos de aplicação, consumo de energia e baixo investimento inicial [2].

Assim, propõe-se o desenvolvimento de uma revisão sistemática da literatura com o objetivo de reunir, avaliar criticamente e conduzir uma síntese dos resultados de múltiplos estudos primários acerca dos fatores que influenciam a produção de juntas soldadas com boas propriedades mecânicas através do processo de soldagem por atrito rotacional, em materiais poliméricos termoplásticos. 


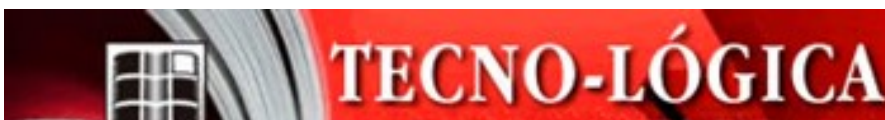

\section{Metodologia}

A pesquisa foi realizada por meio de levantamento bibliográfico nos bancos de dados Science Direct, Portal de Periódicos da Capes e Google Scholar. O critério de busca utilizado foi a presença do termo "Spin Welding" em títulos, resumos ou palavras-chave nos materiais pesquisados. Artigos com foco em materiais metálicos foram excluídos da busca, mantendo apenas estudos com foco em materiais poliméricos.

Inicialmente, foram filtrados somente artigos dos últimos sete (7) anos, porém, a partir da leitura detalhada do material, constatou-se que a maior parte dos autores utilizava como referência artigos clássicos, reconhecidamente relevantes ao tema, os quais foram incluídos na pesquisa. Também foram examinados livros com autores renomados na área de investigação e patentes relativas ao tema.

As referências bibliográficas dos materiais encontrados foram analisadas visando à localização dos artigos primários ou outros materiais relevantes.

A partir da filtragem dos artigos, o critério de aceite para inclusão foi a presença de estudos a respeito dos fatores de influência na resistência das juntas soldadas, os quais foram transcritos, avaliados e confrontados entre si, buscando validar e compreender os fatores percebidos e responder à questão norteadora desta pesquisa.

\section{Resultados e discussões}

\subsection{Soldagem de Polímeros}

No processo de soldagem, calor é aplicado para amolecer (materiais amorfos) ou fundir (materiais semicristalinos) - o material polimérico presente nas superfícies de contato, permitindo a formação de uma junta, através de difusão intermolecular e emaranhamento de cadeias [1-5].

A maior parte dos processos de solda tem em comum cinco etapas distintas: preparação das superfícies, aquecimento, pressão, difusão intermolecular e resfriamento [5]. Entretanto, do ponto de vista físico/químico, o processo passa por quatro (4) estágios distintos: rearranjo das cadeias moleculares nas duas superfícies de solda, espalhamento, interdifusão das cadeias moleculares e emaranhamento (aleatorização) das cadeias [6].

Diversos estudos defendem que os estágios de interdifusão e emaranhamento de cadeias moleculares seriam responsáveis por determinar a resistência da solda [5-7], fenômeno que seria compreendido, pela teoria da reptação, desenvolvida por De Gennes e, posteriormente, por Doi e Edwards, para géis poliméricos, polímeros fundidos e soluções poliméricas concentradas [5].
A teoria de De Gennes assume que, em condições de movimentação restrita pela presença de cadeias vizinhas, as moléculas se deslocam através de movimentos de reptação, tendo sua movimentação limitada pelos pontos de embaraçamento [8]. A Figura 1 ilustra o movimento de reptação proposto por De Gennes:

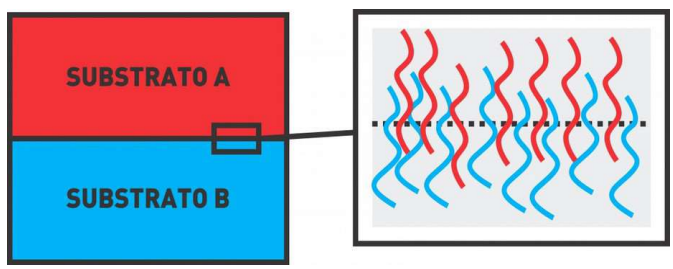

Figura 1 - Movimento de Reptação [9].

Como resultado, a interface desaparece gradualmente por meio de um processo de cura e a resistência mecânica aumenta na interface gradualmente com o decorrer do tempo de contato [9].

Entretanto, nestes modelos são consideradas diversas suposições fundamentais, como superfícies de soldagem completa e intimamente em contato e temperaturas relativamente constantes. Na maior parte das aplicações estas suposições não são corretas, uma vez que, mesmo em superfícies relativamente lisas, picos de aspereza impedem contato íntimo entre as superfícies, sendo tal contato possível somente após o escoamento das mesmas. Ainda, somente experimentos de elevado controle atingem condições constantes de temperatura. Durante a soldagem, estes picos de aspereza amolecem e escoam, preenchendo os espaços intersticiais [1].

Grewell e Benatar [10] estudaram modelos acoplados de temperatura, difusão e escoamento para predição de cura entre superfícies, nos quais propõem um modelo matemático para a predição do espaço entre as superfícies em função do tempo. Segundo eles, uma vez que as interfaces conformam-se uma a outra, elas curam unidas pela difusão de cadeias poliméricas que migram através da interface, tornando-se indistinguíveis do material pré-moldado [1]. A Figura 2 apresenta os detalhes da cura molecular da interface em função do tempo.

Após a cura, ocorre o resfriamento e resolidificação do polímero na superfície de união, na qual as matrizes semicristalinas recristalizam para obter sua microestrutura final. Polímeros amorfos mantêm a orientação molecular previamente induzida, podendo permanecerem distorções ou tensões residuais induzidas termicamente e influenciando na resistência final da peça soldada [5]. 


\section{腈. TECNO-LÓGICA
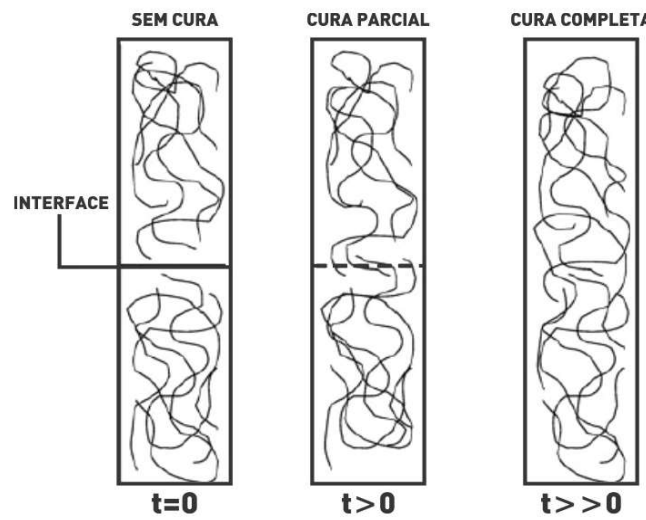

Figura 2 - Cura molecular em função do tempo [1].

\subsection{Soldagem por Fricção}

Os processos de soldagem são comumente categorizados e identificados pelo método de aquecimento usado, sendo divididos em duas categorias gerais: aquecimento interno e aquecimento externo.

Processos com aquecimento mecânico interno baseiamse em princípios básicos similares e possuem diversas vantagens em relação aos demais, entre eles, baixo consumo de energia (uma vez que o calor necessário para a soldagem é proveniente do atrito gerado entre os componentes), tempos de soldagem mais curtos, alta taxa de produção e juntas com bom desempenho mecânico [11].

Existem quatro variações deste processo, em razão de seu movimento mecânico: linear, orbital, angular ou rotacional.

Processos com fricção não linear propiciam linhas de solda com maior resistência, permitindo movimentação nos eixos x e y, ou, ainda, movimento uniforme axial, reduzindo variações processuais em razão da geometria da peça [1].

Oliveira et al. [12] analisaram a soldabilidade de polímeros termoplásticos pela técnica de Soldagem Pontual por Fricção (Friction Spot Welding), por meio da soldagem de placas confeccionadas em polimetilmetacrilato (PMMA). As amostras foram analisadas por microscopia óptica, medições de microdureza Vickers e ensaio de resistência ao cisalhamento.

Considerando que a microdureza média encontrada no material-base foi de $217,8 \pm 3 \mathrm{MPa}$, observou-se que uma ampla região apresentou queda de microdureza na faixa de 5 a $10 \%$, ao que os autores constataram que englobava regiões de polímero amolecido, regiões afastadas ou que tiveram contato com a ferramenta, revelando influência do trabalho mecânico da ferramenta e da alta temperatura.

Para avaliar a resistência ao cisalhamento das juntas, os autores realizaram ensaios com profundidades de penetração de $3,5 \mathrm{~mm}$ e $4 \mathrm{~mm}$, mantendo constantes os parâmetros de RPM (500) e tempo de soldagem (5,5 segundos). As resistências médias encontradas foram de $6,6 \pm 0,6 \mathrm{MPa}(3,5 \mathrm{~mm}$ de penetração) e $8,3 \pm 1,2 \mathrm{MPa}$ (4mm de penetração). Os valores médios superiores para as amostras com maior profundidade originaram-se da maior área de união entre as placas.

Por meio da avaliação da resistência mecânica das peças, comparativamente a placas soldadas por ultrassom, microondas e thermal bonding, o autor constatou que as propriedades mecânicas finais foram superiores para a técnica por fricção.

Strand [13] estudou o efeito da variação dos parâmetros de soldagem (diâmetro do pino, taxa de alimentação, temperatura do calço e tempo de pressão) na microestrutura e propriedades mecânicas de uma junta soldada de polipropileno através do processo de soldagem por fricção (Friction Stir Welding).

As propriedades mecânicas foram ensaiadas com base na norma 2203-5, "Análise de juntas soldadas em formato tipo prato ou tubo: ensaio de dobramento", apresentada pela Associação Alemã de Soldagem (DVS - Deutscher Verband Für Schweissen), que determina normas específicas para ensaios mecânicos em corpos de prova poliméricos soldados. A norma estabelece critérios para aceite e classificação como "boa solda" para alguns métodos de soldagem como gás quente, extrusão e soldagem a laser [14].

Dentre os quatro parâmetros estudados, três apresentaram efeitos estatisticamente significantes no ângulo de dobramento antes da falha: um maior diâmetro do pino, resultando em um aumento do ângulo em 25\%; uma taxa de alimentação de $51 \mathrm{~mm} / \mathrm{mm}$, apresentando um aumento de $13 \%$ em relação à segunda maior e uma temperatura elevada do calço, na faixa de $177{ }^{\circ} \mathrm{C}$, propiciando um aumento de $20 \%$ em relação ao segunda maior $\left(110^{\circ} \mathrm{C}\right)$.

Foram produzidas soldas com resistência à flexão de 80$92 \%$ da resistência do material-base e foram encontrados defeitos e tipos de microestruturas similares em todas as soldas, sendo eles: esferulitos, vazios, trincas, linhas de fluxo, entre outros.

Gonçalves [3], por sua vez, investigou o uso de Soldagem Pontual por Fricção (Friction Spot Welding) na fabricação de juntas pontuais entre chapas de poliamida 6 (PA6) sobrepostas. Os parâmetros de soldagem utilizados, bem como suas variações escolhidas, foram: velocidade de rotação (1000 RPM, 1500 RPM e 2000 RPM), tempo de soldagem ( $5 \mathrm{~s}$, $6 \mathrm{~s}$ e $7 \mathrm{~s})$, profundidade de penetração da ferramenta $(5 \mathrm{~mm}$, $5,5 \mathrm{~mm}$ e $6 \mathrm{~mm})$ e tempo de consolidação $(0,5 \mathrm{~s}, 10,5 \mathrm{~s}$ e $20,5 \mathrm{~s})$. A pressão foi mantida constante em $7,0 \mathrm{kN}$ e o resfriamento, aplicável por meio de fluxo de ar, foi mantido desligado durante todo o teste.

Através da produção de 21 condições diferentes de soldagem e com o uso de técnicas estatísticas, o autor concluiu que os parâmetros velocidade de rotação e tempo de soldagem possuem maior influência sobre a resistência mecânica das 


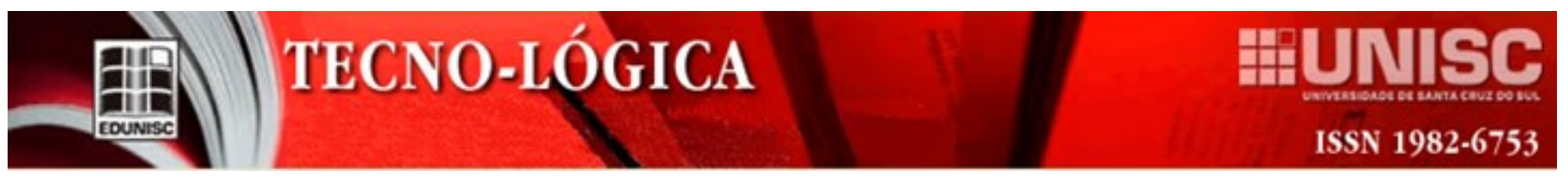

juntas alcançando resistência de $26 \mathrm{MPa}$ por ensaio de cisalhamento sob tração estático. Não houve variação significativa do grau de cristalinidade nas soldas e observou-se um decréscimo de até 7\% na massa molar da poliamida 6 (PA6) em relação ao material de base $(\mathrm{Mv}=41.800 \mathrm{~g} / \mathrm{mol})$.

$\mathrm{O}$ autor justificou este fenômeno segundo o aumento da temperatura de solda (foram obtidas temperaturas de solda de até $275^{\circ} \mathrm{C}$ ), que contribuiu para o consequente aumento da área de soldagem.

\subsection{Soldagem por Atrito Rotacional}

A principal diferença entre as técnicas de soldagem por fricção é a geometria do movimento friccional [1]. No processo de soldagem por atrito rotacional, peças termoplásticas cujas superfícies de união possuem simetria rotacional são friccionadas uma contra a outra sob pressão em um movimento circular unidirecional [2].

Normalmente uma das peças permanece parada enquanto a outra é rotacionada. O calor gerado durante o processo amolece/funde o polímero na interface, formando uma solda por meio de seu resfriamento. A Figura 3 ilustra o processo de soldagem por atrito rotacional:

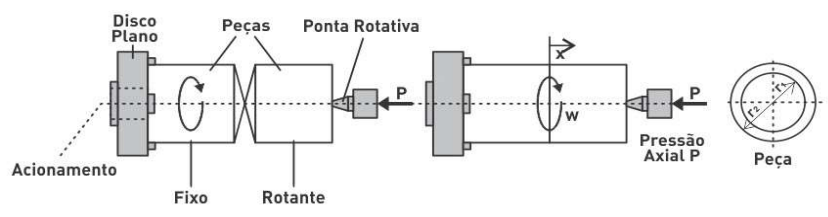

Figura 3 - Soldagem por Atrito Rotacional [7].

O calor necessário para plastificar a zona de soldagem é gerado por fricção externa (entre materiais sólidos) e fricção interna (aquecimento por cisalhamento do material polimérico amolecido/fundido) resultante do movimento entre as superfícies a serem soldadas [15].

Yeh [16] estudou, entre outros métodos, o uso de soldagem por atrito rotacional para aplicações em materiais poliméricos para a indústria médica. Segundo seu estudo, os principais parâmetros processuais para este tipo de solda são o tempo de cura (tempo após a difusão interfacial no qual as peças permanecem estáticas sob pressão constante, propiciando a solidificação do material fundido) e a força/pressão de soldagem. Esta exige maior intensidade do que em outros métodos de soldagem, uma vez que o aquecimento é gerado por meio de fricção.

Ainda, de acordo com o autor, o controle do tempo de aquecimento é fundamental para garantir a presença de material fundido em quantidade suficiente, de forma que o processo de difusão uniforme na interface de junta possa ocorrer sob uma pressão predeterminada, garantindo forte união entre as partes.

Outro aspecto importante é o tempo necessário para cura completa. Muitos estudos têm mostrado que o tempo necessário para a cura é muito menor do que o tempo necessário para o escoamento e espalhamento de material na interface, teoria suportada pelos estudos de Gregwell e Benattar, apresentados anteriormente.

Segundo Yeh [16], como regra geral, em processos de solda que utilizam movimento mecânico/vibração como mecanismo de aquecimento, o desenho da junta tem um papel crítico e sensível na resistência da junta, pois a eficiência da geração de calor é dependente do design.

Rousseau et al [17] apresentaram em sua patente a proposta de novos designs (perfis de solda) para os componentes poliméricos, permitindo o uso de velocidade e pressão relativamente baixas para atingir juntas superiores.

Segundo o autor, componentes com áreas superficiais maiores poderão ser soldados com sucesso com tais designs. Em alguns designs, ao menos um componente polimérico possui uma superfície com diversas características superficiais que são côncavas ou convexas. Em outros designs, o primeiro componente na região de solda possui uma geometria não complementar ao segundo componente, criando assim um avanço progressivo na linha de solda e evitando altas temperaturas que podem danificar o material e a junta de soldagem. Tais designs, adicionalmente, provêm melhora na manutenção de rebarba na superfície de solda.

Sercer e Raos [5] apontam, no entanto, que a criação com sucesso de boas juntas por atrito rotacional também é dependente da condição final das superfícies antes da solda, sofrendo grande influência pela presença de contaminantes como pó, óleos ou graxas. Troughton [2] afirma que aditivos como silicones e outros lubrificantes também reduzem o coeficiente de fricção do material, tornando-o mais difícil de soldar.

Por outro lado, técnicas de soldagem são muito mais tolerantes a fatores como contaminação e variações de materiais entre amostras, comparativamente a outras técnicas de união.

Troughton [2] destaca que o uso de cargas também afeta a resistência da solda, uma vez que quanto maior o percentual de carga presente na composição, menor a quantidade de polímero na interface disponível para a solda. Ainda, uma vez que o processo de solda irá ocorrer entre moléculas do material-base, a resistência máxima que a peça atingirá nesta região será dada pelas propriedades do material puro (sem carga).

\section{Conclusões}

Foi possível constatar a baixa quantidade de estudos aplicados com foco no tema, especialmente nos últimos anos, 


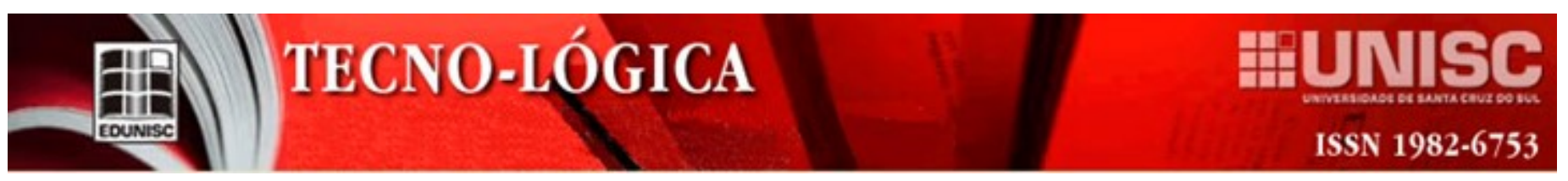

sendo os mesmos concentrados em um pequeno número de autores e com uma abordagem principalmente teórica.

Os estudos avaliados demonstraram que, para técnicas de soldagem por fricção, a etapa de aquecimento é a de maior importância. Temperaturas mais elevadas e maior área de contato produzem juntas melhores propriedades, uma vez que as etapas de interdifusão e emaranhamento de cadeias moleculares provaram ser de grande influência na resistência final das peças soldadas.

Entretanto, os estudos a respeito do comportamento das moléculas durante estas etapas são divergentes e não definitivos, especialmente devido à falta de práticas experimentais reproduzíveis e a falta de maiores estudos que reafirmem ou contraponham ambas as teorias.

A etapa de resfriamento também é de grande importância, sendo a responsável por permitir ou não a consolidação completa da região, sob a pressão predeterminada, e podendo induzir termicamente a criação de tensões ou distorções que afetem a resistência da peça.

Diversos estudos revelaram, ainda, a influência do design da junta para as peças soldadas, condição que pode interferir na eficiência dos demais fatores como, por exemplo, no aquecimento mecânico entre as peças.

Constatou-se, também, que agentes contaminantes e o uso de aditivos ou cargas, mesmo que de maneira reduzida, têm efeito negativo na resistência das peças soldadas.

Como sugestão de desenvolvimento para futuros trabalhos, recomenda-se reavaliar as teorias de De Gennes e Gregwell e Benatar, utilizando uma abordagem mais prática do que teórica, em contraponto aos trabalhos desenvolvidos até o momento. A reavaliação pode colaborar para a construção do conhecimento científico. Sugere-se também avaliar com maior profundidade os perfis de solda e sua influência na resistência e aquecimento das peças soldadas, visando à identificação de melhores designs para cada aplicação.

\section{FACTORS THAT INFLUENCE THE ADHESION AND MECHANICAL STRENGTH OF POLYMER PARTS WELDED BY SPIN WELDING: A LITERATURE REVIEW}

\begin{abstract}
The use of polymeric materials in the industry is constantly increasing, and as a consequence, the joint of several parts is often necessary. Joining methods include mechanical fasteners, adhesives, and polymer welding processes. In the spin welding process, the heat is generated by the friction between parts in relative motion, which melts or soften the materials, providing intermolecular diffusion and chain entanglement. For a better understanding of the phenomena that influence the properties of welded parts, a literature review of data banks such as Science Direct, Portal
\end{abstract}

Periódicos Capes, and Google Scholar, with predefined criteria, was made. It was verified that the mechanical strength was affected mainly by factors such as heating and cooling, the use of suitable weld profiles and materials with appropriate properties for the process, and good surface finishing, with no additives, dust and other contaminants.

Keywords: Welding. Polymers. Spin Welding.

\section{Referências}

[1] GREWELL, D.; BENATAR, A. Welding of plastics: fundamentals and new developments. Intern. Polymer Processing XXII. Munich: Carl Hanser Verlag, 2007.

[2] TROUGHTON, M. J. Handbook of plastics joining: a practical guide. 2. ed. New York: William Andrew Inc., 2008.

[3] GONÇALVES, Joel. Soldagem pontual por fricção (FSPW) de poliamida 6 Dissertação (Mestrado em Ciência e Engenharia de Materiais) - Centro de Ciências Exatas e de Tecnologia, Universidade Federal de São Carlos, São Paulo, 2015.

[4] KINLOCH, A. J. Adhesion and Adhesives Science and Technology. 1. ed. London: Chapman and Hall, 1986.

[5] SERCER, M.; RAOS, P. Encyclopedia of Life Support Systems (EOLSS): Joining of Plastics and Composites. Welding Engineering and Technology, 2006.

[6] WOOL. R.P; O'CONNOR. K.M. A Theory of crack healing in polymers Jornal of Applied Physics. AIP Publishing LLC, 1981.

[7] MICHAELI, Walter; GREIF, Helmut; KAUFMANN, Hans; VOSSEBÜRGER, Franz-Josef. Tecnologia dos Plásticos. 1. ed. Edgard Blücher, 1995.

[8] AKCELRUD, Leni. Fundamentos da ciência dos polímeros. Editora Manole Ltda, 2007.

[9] AWAJA, F. Autohesion of polymers. Polymer, 2016.

[10] GREWELL, D.; BENATAR, A. Coupled Temperature, Diffusion and Squeeze Flow Models for Interfacial Healing Predictions. Annual Technical Conference for the Society of Plastic Engineers Proceedings. Brookfield, Connecticut. 2006

[11] WISE, R. J. Thermal welding of polymers. Woodhead Publishing, 1999.

[12] OLIVEIRA, P. H. F; AMANCIO FILHO, S. T; SANTOS, J. F; HAGE JUNIOR, J. Estudo de Viabilidade da Soldagem de Termoplásticos por "Friction Spot Welding” (FspW). Soldag. Insp. São Paulo, 2012.

[13] STRAND, Seth R. Effects of Friction Stir Welding on Polymer Microstructure. Brigham Young University, 2004.

[14] DVS - Deutscher Verband Für Schweissen Und Verwandte Verfahren E.V. Testing of welded joints of thermoplasticsplates and tubes: Technological Bend Test. DVS, 1999.

[15] TAPPE, P; POTENTE, H. New Results on the Spin Welding of Plastics. Polymer engineering and science, 1989.

[16] YEH, H.J. Overview of welding methods for medical plastics. Woodhead Publishing Limited. USA: 2013. 
[17] ROUSSEAU et al. Spin-welding of polymeric components having large surface area or non-complementary weld interfaces. Patent Application Publication. USA: 2016. 